\title{
SEPTEMBRE 1976
}

58॰ ANNÉE - N• 693
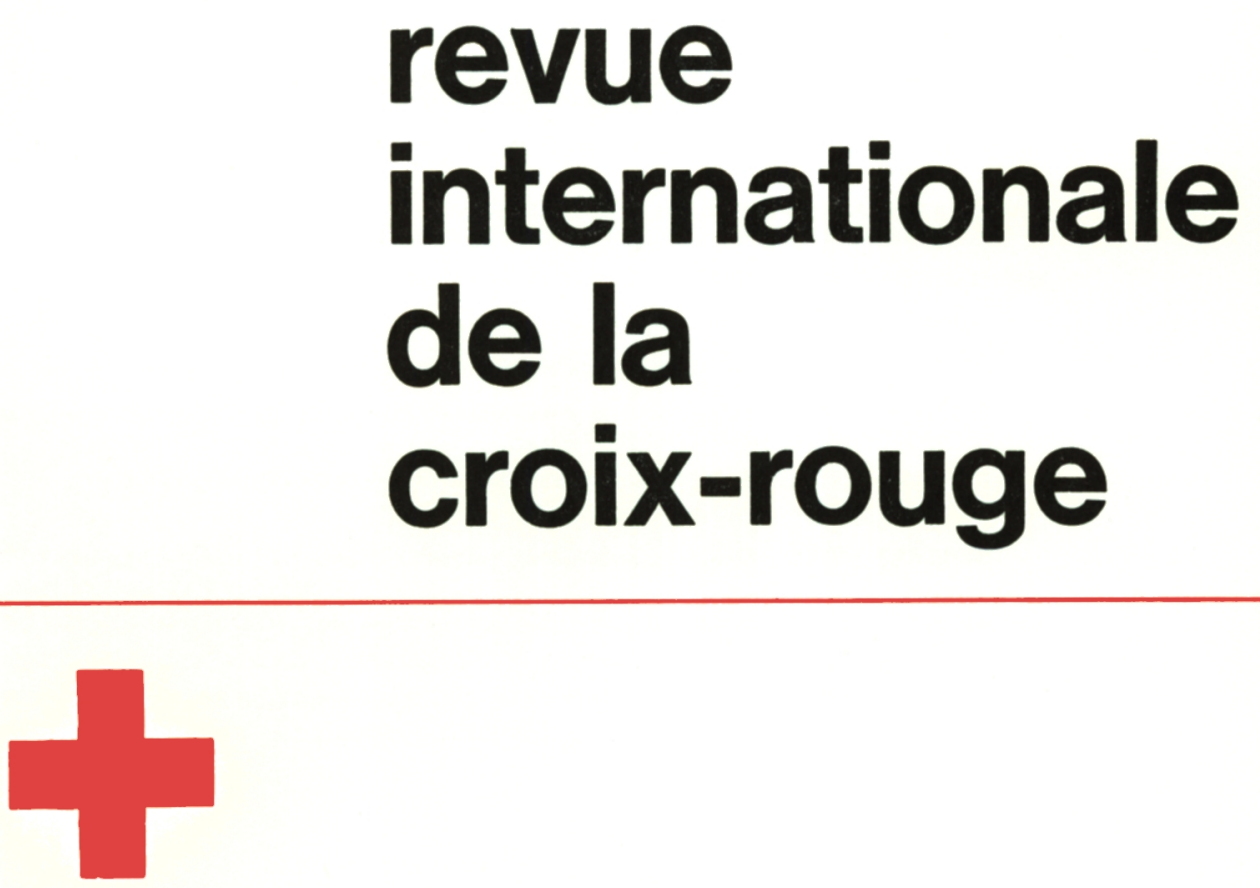

INTER ARMA CARITAS

GENÈVE

COMITÉ INTERNATIONAL DE LA CROIX-ROUGE FONDÉ EN 1863 


\section{COMITÉ INTERNATIONAL DE LA CROIX-ROUGE}

MM. ALEXANDRE HAY, avocat, ancien directeur général de la Banque nationale suisse, président (membre depuis 1975)

JEAN PICTET, docteur en droit, président de la Commission juridique, directeur de l'Institut Henry-Dunant, professeur associé à l'Université, Genève, vice-président (1967)

HARALD HUBER, docteur en droit, juge fédéral, vice-président (1969)

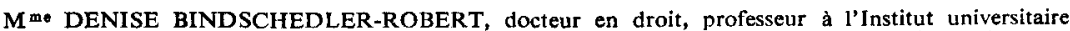
de hautes études internationales, Genève, juge à la Cour européenne des droits de l'homme (1967)

MM. MARCEL A. NAVILLE, licenciè ès lettres, président du CICR de 1969 à 1973 (1967)

JACQUES F. DE ROUGEMONT, docteur en médecine (1967)

ROGER GALLOPIN, docteur en droit, ancien directeur général du CICR (1967)

WALDEMAR JUCKER, docteur en droit, secrétaire de 1'Union syndicale suisse (1967)

VICTOR H. UMBRICHT, docteur en droit, administrateur (1970)

PIERRE MICHELI, licencié en droit, ancien ambassadeur (1971)

GILBERT ETIENNE, professeur à l'Institut universitaire de hautes études internationales et à l'Institut d'études du développement, Genève (1973)

ULRICH MIDDENDORP, docteur en médecine, chef de la clinique chirurgicale de l'Hôpital cantonal, Winterthour (1973)

Mm• MARION BOVEE-ROTHENBACH, diplômée M.S.W. de l'Úniversité de Michigan, maftreassistant à l'Ecole des sciences sociales et politiques de l'Université, Lausanne (1973)

MM. HANS PETER TSCHUDI, docteur en droit, ancien conseiller fédéral (1973)

HENRY HUGUENIN, directeur de banque (1974)

GOTTFRIED DE SMIT, administrateur (1974)

JAKOB BURCKHARDT, docteur en droit, ministre plénipotentiaire, président du Conseil des Ecoles polytechniques fédérales (1975)

THOMAS FLEINER, docteur en droit, professeur à l'Université de Fribourg (1975)

HERBERT LUTHY, docteur en philosophie, professeur d'histoire à l'Université de Baie (1975)

Membres honoraires: $\mathrm{M}^{11}$ LUCIE ODIER, vice-présidente d'honneur; MM. HANS BACHMANN GUILLAUME BORDIER, PAUL CARRY, M Mo MARGUERITE GAUTIER-VAN BERCHEM, MM. ADOLPHE GRAEDEL, EDOUARD DE HALLER, ERIC MARTIN, RODOLFO OLGIATI, MAX PETITPIERRE, PAUL RUEGGER, DIETRICH SCHINDLER, FRÉDÉRIC SIORDET, ALFREDO VANNOTTI.

\section{CONSEIL EXÉCUTIF}

M. ROGER GALLOPIN, président

M. VICTOR H. UMBRICHT, vice-président

$M^{\text {me }}$ DENISE BINDSCHEDLER-ROBERT

M. GILBERT ETIENNE

Dr ULRICH MIDDENDORP

M. JEAN PICTET

M, GOTTFRIED DE SMIT

M. PIERRE MICHELI, membre suppleant 


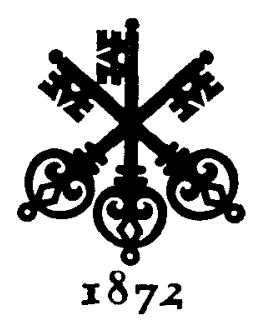

PRÉSENTE SUR LES CINO CONTINENTS, LA GRANDE BANQUE AUX TROIS CLEFS EST À VOTRE DISPOSITION POUR EFFECTUER TOUTES VOS OPÉRATIONS FINANCIĖRES

\section{SOCIÉTÉ DE BANQUE SUISSE}

SCHWEIZERISCHER BANKVEREIN - SOCIETȦ DI BANCA SVIZZERA SWISS BANK CORPORATION

SIËGE DE GENËVE

2, RUE DE LA CONFÉDÉRATION TÉL. 224111

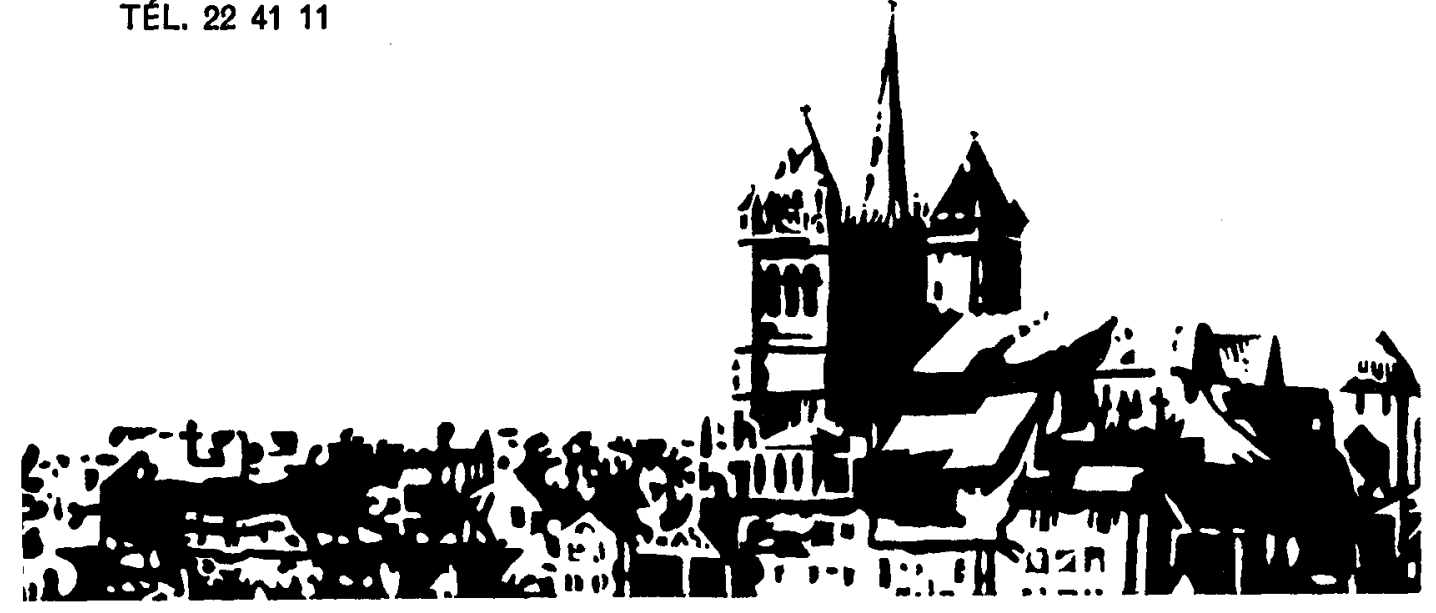




\section{FIDUCIAIRE OFOR S.A.}

Place Saint-Gervais 1, Genève - Tél. 317050

- Expertises, révisions et organisations comptables

Domiciliation et administration de sociétés

Assainissements et liquidations

Interventions et conseils en matière fiscale

\section{JOURNAL DE GENEVE}

deux imprimeries

deux adresses

rue François-Dussaud 20

1211 Genève 8 - Téléphone 431940

rue Général-Dufour 5-7

1211 Genève 11 - Téléphone 280350

TYPO - OFFSET - ROTATIVE 


\section{REVUE INTERNATIONALE DE LA CROIX-ROUGE}

\section{SOMMAIRE}

COMITE INTERNATIONAL DE LA CROIX-ROUGE

\section{DANS LE MONDE DE LA CROIX-ROUGE}

FAITS ET DOCUMENTS

LIVRES ET REVUES
SEPTEMBRE $1976-N^{\circ} 693$

Conférence diplomatique

Résumé des travaux de la troisième session . . . 515

Activités extérieures:

Moyen-Orient - Afrique - Amérique latine -

Asie - URSS . . . . . . . . . 545

Rapport annuel de la Ligue . . . . . . . 552

Cuba ............. 553

Philippines . . . . . . . . . . 556

Enseignement du droit des conflits armés . . . . 559

............. 560 


\section{INTERNATIONAL \\ REVIEW \\ OF THE RED CROSS}

\section{REVISTA INTERNACIONAL DE LA CRUZ ROJA}

\section{EXTRAITS \\ DE LA REVUE}

EN LANGUE ALLEMANDE
Une édition en langue anglaise et une en langue espagnole paraissent chaque mois. Elles sont en principe identiques à l'édition française, et peuvent être obtenues aux mêmes conditions.

Informationsmaterial über die Genfer Abkommen - Die Besucher des IKRK - Hundertjahrfeier des Dänischen Roten Kreuzes - Ausbildung im Kriegsrecht.

Elle est publiée chaque mois par le Comité international de la Croix-Rouge.

17, avenue de la Paix, 1211 Genève (Suisse) - Compte de chèques postaux $12-1767$.

Abonnement un an: Fr. 30,一; le numéro: Fr. 3,-

RÉDACTION: J.-G. LOSSIER

Seuls les textes signés par le Comité international de la Croix-Rouge engagent la responsabilité de celui-ci. 